\title{
Re: Prostate Cancer Treatment in Renal Transplant Recipients: A Systematic Review
}

\author{
Marra G', Dalmasso E', Agnello M'1, Munegato $\mathrm{S}^{1}$, Bosio A', Sedigh 0', Biancone $\mathrm{L}^{2}$, Gontero $\mathrm{P}^{1}$ \\ ${ }^{1}$ University of Studies of Turin, Molinette Hospital, Department of Urology, Turin, Italy \\ ${ }^{2}$ University of Studies of Turin, Molinette Hospital, Department of Nephrology and Renal Transplantation, Turin, Italy \\ BJU Int 2018;121:327-344. doi: 10.1111/bju.14018.
}

\section{EDITORIAL COMMENT}

Prostate cancer ( $\mathrm{PCa}$ ) is the most frequent non-skin cancer observed in male kidney transplant recipients (KTRs) over 50 years of age, probably the rise in its incidence is due to increasing number and age of surviving male KTRs in their mid 50s. This means that an urologist is more likely to evaluate KTRs with PCa more often. However, it is still unknown if the natural history of PCa is changed due to immunosuppression. The aim of this systematic review was to summarize the outcomes of PCa treatment in KTRs. For this purpose, the authors have evaluated 241 patients from 27 retrospective studies published between 1991 and 2016 . In almost $3 / 4$ of cases, PCa was organ-confined and Gleason score was $\leq 6$ in $60 \%$ of patients. Surgery was the most preferred treatment modality, and cancer-specific and overall survival rates were both $96.8 \%$. Functional outcomes, including continence and erectile function, and complications were less frequently reported and were generally similar to those reported for radical prostatectomy in non-KTRs. No treatment-related graft losses have occurred. Immunosuppression and antibiotic schemes were poorly reported and inconsistent. Outcomes of PCa treatment in KTRs seems to be encouraging and do not appear to be inferior to those of non-KTRs. Other treatment modalities preferred in decreasing frequency were radiotherapy, brachytherapy and androgen deprivation treatment. Immunosuppression and antibiotic use were poorly reported and highly variable. This systematic review is important since there is no meta-analysis performed on this topic, however, discreet interpretation of the results is necessary due to lack of strong evidence.

Yarkın Kamil Yakupoğlu, MD

๑Copyright 2018 by the Association of Urological Surgery / Journal of Urological Surgery published by Galenos Publishing House. 